\title{
Waring-Goldbach Problem of Even Powers in Short Intervals
}

\author{
Liqun Hu and Tanhui Zhang $(D)$ \\ Department of Mathematics, Nanchang University, Nanchang 330031, Jiangxi, China \\ Correspondence should be addressed to Tanhui Zhang; zthzth18279187608@163.com \\ Received 13 November 2021; Accepted 29 November 2021; Published 16 December 2021 \\ Academic Editor: Jie Wu
}

Copyright $\odot 2021$ Liqun Hu and Tanhui Zhang. This is an open access article distributed under the Creative Commons Attribution License, which permits unrestricted use, distribution, and reproduction in any medium, provided the original work is properly cited.

In this paper, we study the average behaviour of the representations of $n=p_{1}^{2}+p_{2}^{4}+p_{3}^{4}+p_{4}^{k}$ over short intervals for $k \geq 4$, where $p_{1}, p_{2}, p_{3}, p_{4}$ are prime numbers. This improves the previous results.

\section{Introduction}

Let $n$ and $k_{i}, i=1, \ldots, r$ be integers; the classical Waring-Goldbach problem considers the representation of $n$ in the form

$$
n=p_{1}^{k_{1}}+p_{2}^{k_{2}}+\cdots+p_{r}^{k_{r}} .
$$

This problem has attracted the attention of many authors [1-11]. For sufficiently large integer $N$, let $E(k, N)$ be the exceptional set of expressing positive even integers $n$ up to $N$ in the form

$$
n=p_{1}^{2}+p_{2}^{4}+p_{3}^{4}+p_{4}^{k}
$$

Recently, Feng and Ma [2] established that $E(k, N) \ll N^{1-\theta(k)+\varepsilon}$, here

where

$$
\theta(k)= \begin{cases}\frac{1}{32}, & k=4, \\ \frac{1}{48}, & k=5, \\ \frac{1}{64}, & 6 \leq k \leq 8, \\ \frac{1}{48 x}, & k \geq 9,\end{cases}
$$

$$
x=\left\{\begin{array}{l}
\left\lceil\left(\frac{k}{4}+1-\left[\frac{k}{4}\right]\right) 2\left(\left[\frac{k}{4}\right]-1\right), \quad 9 \leq k \leq 19,\right. \\
\left.\left\lceil\left(\frac{k}{4}-\frac{1}{2}\left[\frac{k}{4}\right]\right)\left(\left[\frac{k}{4}\right]+1\right)\right)\right\rceil, \quad k \geq 20 .
\end{array}\right.
$$

Let

$$
r_{k}(n)=\sum_{n=p_{1}^{2}+p_{2}^{4}+p_{3}^{4}+p_{4}^{k}} \log p_{1} \log p_{2} \log p_{3} \log p_{4} .
$$

In our paper, we use the Hardy-Littlewood circle method and some techniques in the works of Languasco and Zaccagnini [3-8] to improve the results of Feng and Ma by studying the average behaviour of $r_{k}(n)$ over short intervals $[N, N+P], P=o(N)$.

Theorem 1. Let $N$ be a sufficiently large integer and $1 \leq P \leq N, k \geq 4$ be integers. Then, there exists $C=C(\varepsilon) \geq 0$ for every $\varepsilon>0$, such that

$$
\begin{aligned}
\sum_{n=N+1}^{N+P} r_{k}(n)= & \frac{\Gamma(1 / 2)^{2}}{4} P N^{1 / k} \\
& +O_{k}\left(P N^{1 / k} \exp \left(-C\left(\frac{L}{\log L}\right)^{1 / 3}\right)\right)
\end{aligned}
$$

as $N \longrightarrow \infty$, uniformly for $N^{1-5 /(6 k)+\varepsilon} \leq P \leq N^{1-\varepsilon}$. 
Theorem 1 implies that for a sufficiently large integer $N$, every interval $[N, N+P]$ contains the integers which are sums of one prime square, two prime quartics, and one prime power in short intervals, where $N^{1-5 /(6 k)+\varepsilon} \leq P \leq N^{1-\varepsilon}$ for $k \geq 4$. This improves Feng and Ma's results obviously. For example, when $k=4$, the exceptional set $E(4, N) \ll N^{1-5 / 24+\varepsilon}$ in Theorem 1 is smaller than $N^{1-1 / 32+\varepsilon}$ in Feng and Ma's results. Comparing the methods in Feng and Ma's proof process, we replace finite sums with infinite series over primes and get a better result.

\section{Preliminaries and Lemmas}

Let $N$ be a sufficiently large integer, $l \geq 1$ be an integer, $z=1 / N-2 \pi i \alpha, \alpha \varepsilon[-1 / 2,1 / 2], L=\log N, e(\alpha)=e^{2 \pi i \alpha}$,

$$
S_{l}(\alpha)=\sum_{n=1}^{\infty} e^{-n^{l} / N} e\left(n^{l} \alpha\right) \Lambda(n)
$$

and

$$
V_{l}(\alpha)=\sum_{p=2}^{\infty} e^{-p^{l} / N} e\left(p^{l} \alpha\right) \log p
$$

We have

$$
|z|^{-1} \ll \min \left(N,|\alpha|^{-1}\right)
$$

We also set

$$
U(\alpha, P)=\sum_{m=1}^{P} e(m \alpha)
$$

From Montgomery ([11], p. 39), we can find that

$$
|U(\alpha, P)| \leq \min \left(P ;|\alpha|^{-1}\right) \text {. }
$$

Now, we need some lemmas as follows.

Lemma 1 (see [7], Lemma 3). Let $\mu>0, z=1 / N-2 \pi i \alpha$. Then, we have

$$
\int_{-1 / 2}^{1 / 2} z^{-\mu} e(-n \alpha) \mathrm{d} \alpha=\frac{1}{\Gamma(\mu)} e^{-n / N} n^{\mu-1}+O_{\mu}\left(\frac{1}{n}\right),
$$

uniformly for $n \geq 1$.

Lemma 2 (see [7], Lemma 1). $\left|S_{l}(\alpha)-V_{l}(\alpha)\right| \ll{ }_{l} N^{1 /(2 l)}$.

Lemma 3 (see [7], Lemma 4). Let $\varepsilon>0$ be an arbitrarily small positive constant. Then, we have

$$
\int_{-\xi}^{\xi}\left|S_{l}(\alpha)-\frac{\Gamma(1 / l)}{l z^{1 / l}}\right|^{2} \mathrm{~d} \alpha \ll_{l} N^{2 / l-1} \exp \left(-c_{1}\left(\frac{L}{\log L}\right)^{1 / 3}\right),
$$

uniformly for $0 \leq \xi \leq N^{-1+5 /(6 l)-\varepsilon}$. Here, $c_{1}=c_{1}(\varepsilon)$ is a positive constant, which does not depend on $l$.

Lemma 4. Let $\varepsilon>0$ be an arbitrarily small positive constant and $\tau>0$ be a positive constant. Then,

$$
\begin{aligned}
& \int_{-\tau}^{\tau}\left|S_{l}(\alpha)\right|^{2} \mathrm{~d} \alpha \ll_{l}\left(\tau N^{1 / l}+N^{2 / l-1}\right) L^{3}, \\
& \int_{-\tau}^{\tau}\left|S_{l}(\alpha)\right|^{4} \mathrm{~d} \alpha \ll_{l}\left(\tau N^{2 / l}+N^{4 / l-1}\right) N^{\varepsilon}, \\
& \int_{-\tau}^{\tau}\left|V_{l}(\alpha)\right|^{4} \mathrm{~d} \alpha \ll_{l}\left(\tau N^{2 / l}+N^{4 / l-1}\right) N^{\varepsilon} .
\end{aligned}
$$

Proof. This is Lemma 3.4 in [1] and Lemma 6 in [7].

Lemma 5. Let $\varepsilon>0$ be a arbitrarily small positive constant, $c \geq 1$ be a fixed positive constant and $N^{-c} \leq \omega \leq N^{2 / l-1}$. Let $I(\omega):=[-1 / 2,-\omega] \cup[\omega, 1 / 2]$. Then,

$$
\int_{I(w)}\left|S_{l}(\alpha)\right|^{2} \frac{\mathrm{d} \alpha}{|\alpha|} \ll \frac{N^{2 / l-1+\varepsilon}}{\omega} L^{3}+N^{1 / l} L^{4},
$$

$$
\int_{I(w)}\left|S_{l}(\alpha)\right|^{4} \frac{\mathrm{d} \alpha}{|\alpha|} \ll \frac{N^{4 / l-1+\varepsilon}}{\omega}
$$

$$
\int_{I(w)}\left|V_{l}(\alpha)\right|^{4} \frac{\mathrm{d} \alpha}{|\alpha|} \ll \frac{N^{4 / l-1+\varepsilon}}{\omega} .
$$

Proof. This is Lemma 3.5 in [1] and Lemma 7 in [7].

Lemma 6. Let $c_{2}$ be a fixed positive constant; we have

$$
\int_{-1 / 2}^{1 / 2}\left|S_{2}(\alpha) S_{4}(\alpha)^{2}\right|^{2} \mathrm{~d} \alpha \ll N L^{c_{2}} .
$$

Proof. See the proof of Lemma 5 in [7] and Lemma 4.3 in [12] for $k=4$.

\section{Proof of Theorem 1}

Let $\varepsilon>0$ and $P \geq A N^{1-5 /(6 k)+\varepsilon}$ for $k \geq 4$, where $A=N^{2 \varepsilon}$. From equation (5), we can get

$$
\begin{aligned}
& \sum_{n=N+1}^{N+P} e^{-n / N} r_{k}(n) \\
& \quad=\int_{-1 / 2}^{1 / 2} V_{2}(\alpha) V_{4}(\alpha)^{2} V_{k}(\alpha) U(-\alpha, P) e(-N \alpha) \mathrm{d} \alpha .
\end{aligned}
$$

We find it also convenient to set

$$
E_{l}(\alpha)=S_{l}(\alpha)-\frac{\Gamma(1 / l)}{l z^{1 / l}} .
$$

Let $I(A, P)=[-1 / 2,-A / P] \cup[A / P, 1 / 2]$; we can get 


$$
\begin{aligned}
& \sum_{n=N+1}^{N+P} e^{-n / N} r_{k}(n) \\
& =\int_{-A / P}^{A / P} \frac{\Gamma(1 / k) \Gamma(1 / 2)^{2}}{4 k z^{1+1 / k}} U(-\alpha, P) e(-N \alpha) \mathrm{d} \alpha \\
& \quad+\int_{-A / P}^{A / P} \frac{\Gamma(1 / k)}{k z^{1 / k}}\left(S_{2}(\alpha) S_{4}(\alpha)^{2}-\left(\frac{\Gamma(1 / 2)}{2 z^{1 / 2}}\right)^{2}\right) U(-\alpha, P) e(-N \alpha) \mathrm{d} \alpha \\
& \quad+\int_{-A / P}^{A / P} E_{k}(\alpha) S_{2}(\alpha) S_{4}(\alpha)^{2} U(-\alpha, P) e(-N \alpha) \mathrm{d} \alpha \\
& \quad+\int_{-1 / 2}^{1 / 2} V_{k}(\alpha)\left(V_{2}(\alpha) V_{4}(\alpha)^{2}-S_{2}(\alpha) S_{4}(\alpha)^{2}\right) U(-\alpha, P) e(-N \alpha) \mathrm{d} \alpha \\
& \quad+\int_{-1 / 2}^{1 / 2} S_{2}(\alpha) S_{4}(\alpha)^{2}\left(V_{k}(\alpha)-S_{k}(\alpha)\right) U(-\alpha, P) e(-N \alpha) \mathrm{d} \alpha \\
& \quad+\int_{I(A, P)} S_{k}(\alpha) S_{2}(\alpha) S_{4}(\alpha)^{2} U(-\alpha, P) e(-N \alpha) \mathrm{d} \alpha \\
& \quad:=\mathscr{F}_{1}+\mathscr{F}_{2}+\mathscr{F}_{3}+\mathscr{F}_{4}+\mathscr{F}_{5}+\mathscr{F}_{6} .
\end{aligned}
$$

Now, we begin to estimate these terms. First, we estimate $\mathscr{F}_{1}$. By the approximation $e^{-n / N}=e^{-1}+O\left(P N^{-1}\right)$, Lemma 1 , and equations (9) and (11), we can get

$$
\begin{aligned}
\mathscr{F}_{1}= & \frac{\Gamma(1 / k) \Gamma(1 / 2)^{2}}{4 k \Gamma(1+1 / k)} \sum_{n=N+1}^{N+P} n^{1 / k} e^{-n / N}+O_{k}\left(\frac{P}{N}\right) \\
& +O_{k}\left(\int_{A / P}^{1 / 2} \frac{\mathrm{d} \alpha}{\alpha^{2+1 / k}}\right) \\
= & \frac{\Gamma(1 / 2)^{2}}{4 e} P N^{1 / k}+O_{k}\left(P^{2} N^{1 / k-1}+\left(\frac{P}{A}\right)^{1+(1 / k)}+N^{(1 / k)}\right) .
\end{aligned}
$$

Next, we estimate $\mathscr{F}_{2}$.

$$
\begin{aligned}
& \int_{-A / P}^{A / P} \frac{\Gamma(1 / k)}{k z^{1 / k}}\left(S_{2}(\alpha) S_{4}(\alpha)^{2}-\left(\frac{\Gamma(1 / 2)}{2 z^{1 / 2}}\right)^{2}\right) U(-\alpha, P) e(-N \alpha) \mathrm{d} \alpha \\
& =\int_{-A / P}^{A / P} \frac{\Gamma(1 / k)}{k z^{1 / k}}\left(S_{2}(\alpha)^{2}-\frac{\Gamma(1 / 2)^{2}}{4 z}\right) U(-\alpha, P) e(-N \alpha) \mathrm{d} \alpha \\
& \quad+\int_{-A / P}^{A / P} \frac{\Gamma(1 / k)}{k z^{1 / k}}\left(S_{2}(\alpha) S_{4}(\alpha)^{2}-S_{2}(\alpha)^{2}\right) U(-\alpha, P) e(-N \alpha) \mathrm{d} \alpha \\
& =\mathrm{E}_{1}+\mathrm{E}_{2} .
\end{aligned}
$$

From $S_{2}(\alpha) \ll N^{1 / 2}$ and $f^{2}-g^{2}=2 f(f-g)-(f-g)^{2}$, we can get

$$
\begin{aligned}
\mathrm{E}_{1} \ll{ }_{k} N^{1 / 2} \int_{-A / P}^{A / P}\left|E_{2}(\alpha)\right| \frac{|U(-\alpha, P)|}{|z|^{1 / k}} d \alpha \\
\quad+\int_{-A / P}^{A / P}\left|E_{2}(\alpha)^{2}\right| \frac{|U(-\alpha, P)|}{|z|^{1 / k}} \mathrm{~d} \alpha \\
:=\mathrm{E}_{11}+\mathrm{E}_{12} .
\end{aligned}
$$

By Lemma 3 and equation (11), for every $\varepsilon>0$, then there exists $c_{1}=c_{1}(\varepsilon)>0$ such that

$$
\begin{aligned}
\mathrm{E}_{12} & \ll{ }_{k} P N^{1 / k}\left(\int_{-A / P}^{A / P}\left|E_{2}(\alpha)\right|^{2} \mathrm{~d} \alpha\right) \\
& \ll{ }_{k} P N^{1 / k} \exp \left(-c_{1}\left(\frac{L}{\log L}\right)^{1 / 3}\right),
\end{aligned}
$$

provided that $P \geq A N^{1-5 /(6 k)+\varepsilon}$ for $k \geq 4$. By Lemma 3, equation (11), and the Cauchy-Schwarz inequality, we obtain that

$$
\begin{gathered}
\mathscr{E}_{11} \ll_{k} N^{1 / 2}\left(\int_{-A / P}^{A / P}\left|E_{2}(\alpha)\right|^{2} \mathrm{~d} \alpha\right)^{1 / 2} P\left(\int_{-A / P}^{A / P} \frac{\mathrm{d} \alpha}{|z|^{2 / k}}\right)^{1 / 2} \\
\ll{ }_{k} P N^{1 / 2} \exp \left(-\frac{c_{1}}{2}\left(\frac{L}{\log L}\right)^{1 / 3}\right) \\
\cdot\left(\int_{-1 / N}^{1 / N} N^{2 / k} \mathrm{~d} \alpha+\int_{1 / N}^{A / P} \frac{\mathrm{d} \alpha}{|\alpha|^{2 / k}}\right)^{1 / 2} \\
\ll{ }_{k} P N^{1 / k} \exp \left(-\frac{c_{1}}{2}\left(\frac{L}{\log L}\right)^{1 / 3}\right),
\end{gathered}
$$


provided that $P \geq A N^{1-5 /(6 k)+\varepsilon}$ for $k \geq 4$. Hence, by equations (23) and (24), for every $\varepsilon>0$, then there exists $c_{1}=c_{1}(\varepsilon)>0$ such that

$$
\mathrm{E}_{1} \ll_{k} P N^{1 / k} \exp \left(-\frac{c_{1}}{2}\left(\frac{L}{\log L}\right)^{1 / 3}\right),
$$

provided that $P \geq A N^{1-5 /(6 k)+\varepsilon}$ for $k \geq 4$.

$$
\begin{aligned}
\mathrm{E}_{2}= & \int_{-A / P}^{A / P} \frac{\Gamma(1 / k)}{k z^{1 / k}}\left(S_{2}(\alpha) S_{4}(\alpha)^{2}-S_{2}(\alpha)^{2}\right) \\
& \cdot U(-\alpha, P) e(-N \alpha) d \alpha \\
= & \mathrm{E}_{21}+\mathrm{E}_{22} .
\end{aligned}
$$

By Lemma 4, equation (11), and $S_{k}(\alpha) \ll_{k} N^{1 / k}$, we have

$$
\begin{gathered}
\mathrm{E}_{21} \ll{ }_{k} N^{1 / k+1 / 2}\left(\int_{-A / P}^{A / P}\left|S_{4}(\alpha)\right|^{2}|U(-\alpha, P)| \mathrm{d} \alpha\right) \\
\ll{ }_{k} N^{1 / k+1 / 2}\left(\int_{-A / P}^{A / P}\left|S_{4}(\alpha)\right|^{4} \mathrm{~d} \alpha\right)^{1 / 2} \\
\cdot\left(\int_{-1 / P}^{1 / P} P^{2} \mathrm{~d} \alpha+\int_{1 / P}^{A / P}|\alpha|^{-2} \mathrm{~d} \alpha\right)^{1 / 2} \\
\ll{ }_{k} N^{1 / k+1 / 2}\left(\frac{A}{P} N^{1 / 2+\varepsilon}\right)^{1 / 2}\left(P+\frac{P}{A}\right)^{1 / 2} \\
\ll{ }_{k} A^{1 / 2} N^{1 / k+3 / 4+\varepsilon}
\end{gathered}
$$

and

$$
\begin{aligned}
\mathrm{E}_{22} & \ll{ }_{k} N^{1 / k} \int_{-\mathrm{A} / P}^{A / P}\left|S_{2}(\alpha)\right|^{2}|U(-\alpha, P)| \mathrm{d} \alpha \\
& \ll{ }_{k} P N^{1 / k}\left(\int_{-A / P}^{A / P}\left|S_{2}(\alpha)\right|^{4} \mathrm{~d} \alpha\right)^{1 / 2}\left(\int_{-A / P}^{A / P} d \alpha\right)^{1 / 2} \\
& \ll{ }_{k} P N^{1 / k}\left(\frac{A}{P} N^{1+\varepsilon}\right)^{1 / 2}\left(\frac{A}{P}\right)^{1 / 2} \\
& \ll{ }_{k} A N^{1 / k+1 / 2+\varepsilon} .
\end{aligned}
$$

Hence, by equations (27) and (28), we have

$$
\begin{aligned}
\mathrm{E}_{2} & \ll{ }_{k} A N^{1 / k+1 / 2+\varepsilon}+A^{1 / 2} N^{1 / k+3 / 4+\varepsilon} \\
& \ll{ }_{k} P N^{1 / k} \exp \left(-\frac{c_{1}}{2}\left(\frac{L}{\log L}\right)^{1 / 3}\right),
\end{aligned}
$$

provided that $P \geq A N^{1-5 /(6 k)+\varepsilon}$ for $k \geq 4$. Thus, from equations (25) and (29), for every $\varepsilon>0$, there exists $c_{1}=c_{1}(\varepsilon)>0$ such that

$$
\mathscr{F}_{2} \ll_{k} P N^{1 / k} \exp \left(-\frac{c_{1}}{2}\left(\frac{L}{\log L}\right)^{1 / 3}\right),
$$

provided that $P \geq A N^{1-5 /(6 k)+\varepsilon}$ for $k \geq 4$

Now, we estimate $\mathscr{F}_{3}$. By equations (11) and (19), Lemmas 3 and 6, and the Cauchy-Schwarz inequality, we obtain that for every $\varepsilon>0$, there exists $c_{1}=c_{1}(\varepsilon)>0$ such that

$$
\begin{aligned}
\mathscr{F}_{3} & \ll{ }_{k} P\left(\int_{-1 / 2}^{1 / 2}\left|S_{2}(\alpha) S_{4}(\alpha)^{2}\right|^{2} \mathrm{~d} \alpha\right)^{1 / 2}\left(\int_{-A / P}^{A / P}\left|E_{k}(\alpha)\right|^{2} \mathrm{~d} \alpha\right)^{1 / 2} \\
& { }_{k} P N^{1 / 2} L^{c_{2} / 2}\left(\int_{-A / P}^{A / P}\left|E_{k}(\alpha)\right|^{2} \mathrm{~d} \alpha\right)^{1 / 2} \\
& \ll_{k} P N^{1 / k} \exp \left(-\frac{c_{1}}{2}\left(\frac{L}{\log L}\right)^{1 / 3}\right),
\end{aligned}
$$

provided that $P \geq A N^{1-5 /(6 k)+\varepsilon}$ for $k \geq 4$.

Next, we estimate $\mathscr{F}_{4}$.

$$
\begin{aligned}
\mathscr{F}_{4}= & \int_{-1 / 2}^{1 / 2} V_{k}(\alpha)\left(V_{2}(\alpha) V_{4}(\alpha)^{2}-S_{2}(\alpha) S_{4}(\alpha)^{2}\right) U(-\alpha, P) e(-N \alpha) \mathrm{d} \alpha \\
= & \int_{-1 / 2}^{1 / 2} V_{k}(\alpha) V_{4}(\alpha)^{2}\left(V_{2}(\alpha)-S_{2}(\alpha)\right) U(-\alpha, P) e(-N \alpha) \mathrm{d} \alpha \\
& +\int_{-1 / 2}^{1 / 2} V_{k}(\alpha) S_{2}(\alpha)\left(V_{4}(\alpha)^{2}-S_{4}(\alpha)^{2}\right) U(-\alpha, P) e(-N \alpha) \mathrm{d} \alpha \\
:= & \mathscr{U}_{1}+\mathscr{U}_{2} .
\end{aligned}
$$

By Lemma 2 and $V_{k}(\alpha) \ll_{k} N^{1 / k}$, we obtain that 


$$
\begin{aligned}
\mathscr{U}_{2} & =\int_{-1 / 2}^{1 / 2} V_{k}(\alpha)\left(V_{4}(\alpha)-S_{4}(\alpha)\right) S_{2}(\alpha)\left(V_{4}(\alpha)+S_{4}(\alpha)\right) U(-\alpha, P) e(-N \alpha) \mathrm{d} \alpha \\
& \ll{ }_{k} N^{1 / k+1 / 8} \int_{-1 / 2}^{1 / 2}\left(S_{2}(\alpha) V_{4}(\alpha)+S_{2}(\alpha) S_{4}(\alpha)\right) U(-\alpha, P) e(-N \alpha) \mathrm{d} \alpha \\
& :=N^{1 / k+1 / 8}\left(\mathscr{J}_{1}+\mathscr{J}_{2}\right) .
\end{aligned}
$$

By Lemmas 4 and 5, equation (11), and the Cauchy-Schwarz inequality, we have

$$
\begin{aligned}
\mathscr{J}_{1}= & \int_{-1 / 2}^{1 / 2} S_{2}(\alpha) V_{4}(\alpha) U(-\alpha, P) e(-N \alpha) \mathrm{d} \alpha \\
\ll & P \int_{-1 / P}^{1 / P}\left|S_{2}(\alpha) \| V_{4}(\alpha)\right| \mathrm{d} \alpha+\int_{I(1, P)} \frac{\left|S_{2}(\alpha) \| V_{4}(\alpha)\right|}{|\alpha|} \mathrm{d} \alpha \\
\ll & P\left(\int_{-1 / P}^{1 / P}\left|S_{2}(\alpha)\right|^{2} \mathrm{~d} \alpha\right)^{1 / 2}\left(\int_{-1 / P}^{1 / P}\left|V_{4}(\alpha)\right|^{2} \mathrm{~d} \alpha\right)^{1 / 2} \\
& +\left(\int_{I(1, P)} \frac{\left|S_{2}(\alpha)\right|^{2}}{|\alpha|} \mathrm{d} \alpha\right)^{1 / 2}\left(\int_{I(1, P)} \frac{\left|V_{4}(\alpha)\right|^{2}}{|\alpha|} \mathrm{d} \alpha\right)^{1 / 2} \\
\ll & P\left(\frac{1}{P} N^{1 / 2}+1\right)^{1 / 2} L^{3 / 2}\left(\left.\int_{-1 / P}^{1 / P} V_{4}(\alpha)\right|^{4} \mathrm{~d} \alpha\right)^{1 / 4}\left(\int_{-1 / P}^{1 / P} \mathrm{~d} \alpha\right)^{1 / 4} \\
& +\left(N^{1 / 2} L^{4}+P L^{3}\right)^{1 / 2}\left(\int_{I(1, P)} \frac{\left|V_{4}(\alpha)\right|^{4}}{|\alpha|} \mathrm{d} \alpha\right)^{1 / 4}\left(\int_{I(1, P)} \frac{1}{|\alpha|} \mathrm{d} \alpha\right)^{1 / 4} \\
& \ll P^{3 / 4} N^{\varepsilon / 4} L^{3 / 2}+N^{1 / 4+\varepsilon / 4} P^{1 / 4} L^{9 / 4} \\
& \ll P^{3 / 4} N^{\varepsilon / 4} L^{3 / 2},
\end{aligned}
$$

provided that $P \geq A N^{1-5 /(6 k)+\varepsilon}$ for $k \geq 4$.

Similarly, we also have

$$
\begin{aligned}
\mathscr{J}_{2}= & \int_{-1 / 2}^{1 / 2} S_{2}(\alpha) S_{4}(\alpha) U(-\alpha, P) e(-N \alpha) \mathrm{d} \alpha \\
\ll & P \int_{-1 / P}^{1 / P}\left|S_{2}(\alpha) \| S_{4}(\alpha)\right| \mathrm{d} \alpha+\int_{I(1, P)} \frac{\left|S_{2}(\alpha)\right|\left|S_{4}(\alpha)\right|}{|\alpha|} \mathrm{d} \alpha \\
& \ll P\left(\int_{-1 / P}^{1 / P}\left|S_{2}(\alpha)\right|^{2} \mathrm{~d} \alpha\right)^{1 / 2}\left(\int_{-1 / P}^{1 / P}\left|S_{4}(\alpha)\right|^{2} \mathrm{~d} \alpha\right)^{1 / 2} \\
& +\left(\int_{I(1, P)} \frac{\left|S_{2}(\alpha)\right|^{2}}{|\alpha|} d \alpha\right)^{1 / 2}\left(\int_{I(1, P)} \frac{\left|S_{4}(\alpha)\right|^{2}}{|\alpha|} d \alpha\right)^{1 / 2} \\
\ll & P^{3 / 4} N^{\varepsilon / 4} L^{3 / 2},
\end{aligned}
$$

provided that $P \geq A N^{1-5 /(6 k)+\varepsilon}$ for $k \geq 4$.
Hence, by equations (34) and (35), we have

$$
U_{2} \ll{ }_{k} P^{3 / 4} N^{1 / k+1 / 8+\varepsilon},
$$

provided that $P \geq A N^{1-5 /(6 k)+\varepsilon}$ for $k \geq 4$.

By Lemma 2, equation (11), and $V_{k}(\alpha) \ll_{k} N^{1 / k}$, we have

$$
\begin{aligned}
\mathscr{U}_{1} & =\int_{-1 / 2}^{1 / 2} V_{k}(\alpha) V_{4}(\alpha)^{2}\left(V_{2}(\alpha)-S_{2}(\alpha)\right) U(-\alpha, P) e(-N \alpha) \mathrm{d} \alpha \\
& \ll{ }_{k} N^{1 / k+1 / 4}\left(\int_{-1 / 2}^{1 / 2}\left|V_{4}(\alpha)\right|^{2}|U(-\alpha, P)| \mathrm{d} \alpha\right) \\
& \ll{ }_{k} N^{1 / k+1 / 4}\left(P \int_{-1 / P}^{1 / P}\left|V_{4}(\alpha)\right|^{2} \mathrm{~d} \alpha+\int_{I(1, P)} \frac{\left|V_{4}(\alpha)\right|^{2}}{|\alpha|} \mathrm{d} \alpha\right) \\
& :=N^{1 / k+1 / 4}\left(\mathscr{M}_{1}+\mathscr{M}_{2}\right) .
\end{aligned}
$$

By Lemmas 4 and 5 and the Cauchy-Schwarz inequality, we have 


$$
\mathscr{M}_{1} \ll P\left(\int_{-1 / P}^{1 / P}\left|V_{4}(\alpha)\right|^{4} \mathrm{~d} \alpha\right)^{1 / 2}\left(\int_{-1 / P}^{1 / P} \mathrm{~d} \alpha\right)^{1 / 2} \ll P^{1 / 2} N^{\varepsilon / 2},
$$

and

$$
\mathscr{M}_{2} \ll\left(\int_{I(1, P)} \frac{\left|V_{k}(\alpha)\right|^{4}}{|\alpha|} \mathrm{d} \alpha\right)^{1 / 2}\left(\int_{I(1, P)} \frac{1}{|\alpha|} \mathrm{d} \alpha\right)^{1 / 2} \ll P^{1 / 2} N^{\varepsilon},
$$

provided that $P \geq A N^{1-5 /(6 k)+\varepsilon}$ for $k \geq 4$. Then, we have

$$
\mathcal{U}_{1} \ll_{k} P^{1 / 2} N^{1 / k+1 / 4+\varepsilon},
$$

provided that $P \geq A N^{1-5 /(6 k)+\varepsilon}$ for $k \geq 4$.

Hence, by equations (36) and (40), we have

$$
\begin{aligned}
\mathscr{F}_{4} & \ll{ }_{k} P^{1 / 2} N^{1 / k+1 / 4+\varepsilon}+P^{3 / 4} N^{1 / k+1 / 8+\varepsilon} \\
& \ll{ }_{k} P N^{1 / k} \exp \left(-\frac{c_{1}}{2}\left(\frac{L}{\log L}\right)^{1 / 3}\right),
\end{aligned}
$$

provided that $P \geq A N^{1-5 /(6 k)+\varepsilon}$ for $k \geq 4$.

Now, we estimate $\mathscr{F}_{5}$. By Lemmas 4 and 5 , equation (11), and a partial integration, we have

$$
\begin{aligned}
\mathscr{F}_{5} & \ll{ }_{k} P N^{1 / 2 k+1 / 2}\left(\int_{-1 / P}^{1 / P}\left|S_{4}(\alpha)\right|^{2} \mathrm{~d} \alpha\right) \\
& +N^{1 / 2 k+1 / 2}\left(\int_{I(1, P)} \frac{\left|S_{4}(\alpha)\right|^{2}}{|\alpha|} \mathrm{d} \alpha\right) \\
& \ll{ }_{k} P N^{1 / 2 k+1 / 2}\left(\frac{N^{1 / 4}}{P}+N^{-1 / 2}\right) L^{3}+\left(P N^{\varepsilon}\right) N^{1 / 2 k+1 / 2} \\
& \ll{ }_{k} P N^{1 / k} \exp \left(-\frac{c_{1}}{2}\left(\frac{L}{\log L}\right)^{1 / 3}\right),
\end{aligned}
$$

provided that $P \geq A N^{1-5 /(6 k)+\varepsilon}$ for $k \geq 4$.

Finally, we estimate $\mathscr{F}_{6}$. Clearly, by Lemma 5 , equation (11), and Cauchy-Schwarz inequality, we have

$$
\begin{aligned}
\mathscr{F}_{6} & \ll{ }_{k} N^{1 / k}\left(\int_{I(A, P)} \frac{\left|S_{2}(\alpha)\right|^{2}}{|\alpha|} \mathrm{d} \alpha\right)^{1 / 2}\left(\int_{I(A, P)} \frac{\left|S_{4}(\alpha)\right|^{4}}{|\alpha|} d \alpha\right)^{1 / 2} \\
& \ll{ }_{k} N^{1 / k}\left(N^{1 / 2} L^{4}+\frac{P}{A} L^{3}\right)^{1 / 2}\left(N^{\varepsilon} \frac{P}{A}\right)^{1 / 2} \\
& \ll{ }_{k} N^{1 / k}\left(N^{1 / 2+\varepsilon} L^{4}\left(\frac{P}{A}\right)+\left(\frac{P}{A}\right)^{2} N^{\varepsilon} L^{3}\right)^{1 / 2} \\
& \ll{ }_{k} \frac{P}{A} N^{1 / k+\varepsilon / 2} L^{2 / 3} \\
& \ll{ }_{k} P N^{1 / k} \exp \left(-\frac{c_{1}}{2}\left(\frac{L}{\log L}\right)^{1 / 3}\right),
\end{aligned}
$$

provided that $P \geq A N^{1-5 /(6 k)+\varepsilon}$ for $k \geq 4$.

Completion of the proof. Let $k \geq 4$. By equations (19) $-(37)$ and (40)-(43), we obtain that, for every $\varepsilon>0$, there exists $C=C(\varepsilon)>0$, such that

$$
\begin{aligned}
\sum_{n=N+1}^{N+P} e^{-n / N} r_{k}(n)= & \frac{\Gamma(1 / 4)^{2}}{4 e} P N^{1 / k} \\
& +O_{k}\left(P N^{1 / k} \exp \left(-C\left(\frac{L}{\log L}\right)^{1 / 3}\right)\right),
\end{aligned}
$$

provided that $P \geq A N^{1-5 /(6 k)+\varepsilon}$ with $A=N^{2 \varepsilon}$. From $e^{-n / N}=e^{-1}+O\left(P N^{-1}\right)$, for $n \in[N+1, N+P], 1 \leq P \leq N$ and every $\varepsilon>0$, there exists $C=C(\varepsilon)>0$, such that

$$
\begin{aligned}
e^{-1} \sum_{n=N+1}^{N+P} r_{k}(n)= & \frac{\Gamma(1 / 2)^{2}}{4 e} P N^{1 / k} \\
& +O_{k}\left(P N^{1 / k} \exp \left(-C\left(\frac{L}{\log L}\right)^{1 / 3}\right)\right) \\
& +O_{k}\left(\frac{P}{N} \sum_{n=N+1}^{N+P} r_{k}(n)\right),
\end{aligned}
$$

provided that $N^{1-5 / 6 k+\varepsilon} \leq P \leq N^{1-\varepsilon}$ for $k \geq 4$. Using $e \leq e^{n / N} \leq e^{2}$ and equation (44), the last error term is $\ll_{k} P^{2} N^{1 / k-1}$. Thus, we have

$$
\begin{aligned}
\sum_{n=N+1}^{N+P} r_{k}(n)= & \frac{\Gamma(1 / 2)^{2}}{4} P N^{1 / k} \\
& +O_{k}\left(P N^{1 / k} \exp \left(-C\left(\frac{L}{\log L}\right)^{1 / 3}\right)\right),
\end{aligned}
$$

uniformly for $N^{1-5 / 6 k+\varepsilon} \leq P \leq N^{1-\varepsilon}$. Now, Theorem 1 follows.

\section{Data Availability}

The data used to support this study are available upon request from the corresponding author.

\section{Conflicts of Interest}

The authors declare that they have no conflicts of interest.

\section{Acknowledgments}

This work was supported by the Natural Science Foundation of Jiangxi Province for Distinguished Young Scholars (Grant no. 20212ACB211007) and Natural Science Foundation of China (Grant no. 11761048). 


\section{References}

[1] M. Cantarini, A. Gambini, A. Languasco, and A. Zaccagnini, "On an average ternary problem with prime powers," The Ramanujan Journal, vol. 53, no. 1, pp. 155-166, 2020.

[2] Z. Feng and J. Ma, "Waring-goldbach problem for unlike powers," 2019, https://arxiv.org/abs/1907.11918v1.

[3] A. Languasco and A. Zaccagnini, "Sum of one prime and two squares of primes in short intervals," Journal of Number Theory, vol. 159, pp. 1945-1960, 2016.

[4] A. Languasco and A. Zaccagnini, "Short intervals asymptotic formulae for binary problems with primes and powers, II: density 1," Monatshefte für Mathematik, vol. 181, no. 2, pp. 419-435, 2016.

[5] A. Languasco and A. Zaccagnini, "Short intervals asymptotic formulae for binary problems with primes and powers, I: density 3/2," The Ramanujan Journal, vol. 42, no. 2, pp. 371-383, 2017.

[6] A. Languasco and A. Zaccagnini, "Short intervals asymptotic formulae for binary problems with prime powers," Journal de Théorie des Nombres de Bordeaux, vol. 30, no. 2, pp. 609-635, 2018.

[7] A. Languasco and A. Zaccagnini, "Sums of four prime cubes in short intervals," Acta Mathematica Hungarica, vol. 159, no. 1, pp. 150-163, 2019.

[8] A. Languasco and A. Zaccagnini, "Short intervals asymptotic formulae for binary problems with prime powers, II," Journal of the Australian Mathematical Society, vol. 109, no. 3, pp. 351-370, 2020.

[9] H. F. Liu and J. Huang, "Diophantine approximation with mixed powers of primes, Taiwan," Jurnal Matematika, vol. 23, pp. 1073-1090, 2019.

[10] H. Liu, "Diophantine approximation with one prime, two squares of primes and powers of two," The Ramanujan Journal, vol. 51, no. 1, pp. 85-97, 2020.

[11] H. L. Montgomery, "Ten lectures on the interface between analytic number theory and harmonic analysis," CBMS Regional Conference Series in Mathematics, Vol. 84, American Mathematical Society, , Providence, RI, USA, 1994.

[12] L. Zhao, "On unequal powers of primes and powers of 2," Acta Mathematica Hungarica, vol. 146, no. 2, pp. 405-420, 2015. 